Editorial

\title{
Incontinencia urinaria de esfuerzo femenina: aproximación racional a su diagnóstico y manejo
}

\section{Female Stress Urinary Incontinence: A Rational Approach to its Diagnosis and Management}

\author{
Mariana González La Rotta ${ }^{1} \quad$ Alejandra Bravo-Balado ${ }^{1}$ Anamaria Ramos ${ }^{1}$ Mauricio Plata ${ }^{1}$ \\ ${ }^{1}$ Departamento de Urología, Hospital Universitario Fundación Santa \\ Fe de Bogotá y Facultad de Medicina, Universidad de los Andes, \\ Bogotá D.C., Colombia \\ Urol Colomb 2018;27:115-125.
}

\section{Introducción}

La incontinencia urinaria de esfuerzo (IUE) femenina es una entidad de alta prevalencia que altera la calidad de vida (QoL) de manera significativa, por lo cual la concientización de la comunidad, tanto general como médica, en cuanto a su prevención, diagnóstico y manejo son fundamentales, pudiendo llevar a una disminución de la carga de enfermedad y los costos que derivan de su tratamiento. Esta revisión tiene como objetivo servir de actualización en la determinación de las estrategias diagnósticas útiles para el estudio de la IUE así como un manejo racional basado en la mejor evidencia disponible.

\section{Definiciones y epidemiología}

La incontinencia urinaria (IU), se considera un síntoma cuando es referido por el paciente, un signo al ser un hallazgo al examen físico (EF), o una condición dado su significado patológico cuando es identificada a través de alguna prueba diagnóstica. Esta es definida por la Sociedad internacional de Continencia (ICS) como la pérdida involuntaria de orina. ${ }^{1}$ Por su parte, la IUE es la pérdida involuntaria de orina referida por el paciente asociada a maniobras de esfuerzo.

A nivel mundial, la IU afecta el $17-45 \%$ de mujeres adultas. ${ }^{2}$ El $48 \%$ de los casos corresponden a IUE, el $17 \%$ a incontinencia urinaria de urgencia (IUU) y el resto a incontinencia mixta (IUM). ${ }^{3}$ En Latinoamérica, según Guarisi y cols., la prevalencia en Brasil es del 34,8\%. ${ }^{4}$

En un reciente estudio en Colombia por Plata y cols., se determinó que la prevalencia de IUE en mujeres por encima de los 18 años es del $13,7 \%$, siendo mayor $(24,2 \%)$ en el grupo de mujeres mayores de 65 años. ${ }^{5}$

Por su elevado costo financiero y el impacto en el bienestar social y QoL, esta patología debe considerarse un problema de salud pública. En cuanto al efecto de la IU en la QoL, se ha encontrado que altera múltiples esferas, entre ellas, la función sexual. El 34,6\% de las mujeres con IU tienen disminución del deseo sexual, mientras que el 29,3\% tienen disfunción orgásmica. ${ }^{6}$ De manera similar, un estudio realizado en Colombia encontró que las mujeres con IUE tienen menor deseo sexual (66,7\% versus $82 \%$ ), menor excitación (56,3\% versus $72,9 \%$ ) y menor satisfacción sexual (68,8\% versus $87,2 \%)$. Además, las mujeres colombianas con IUE tienen más tasas de depresión (35,1\% versus $16,8 \%$ ) y ansiedad (27,6\% versus $14,6 \%$ ) que las mujeres que no sufren de esa condición (datos no publicados).

Uno de los principales factores de riesgo de IUE, es el antecedente de parto vaginal. Aunque muchas mujeres desarrollan IU durante el embarazo, a los 3 meses del parto, menos del $1 \%$ de las mujeres persisten con síntomas de IUE. ${ }^{7} \mathrm{El}$ riesgo aumenta hasta 1,6 veces en mujeres con antecedente familiar de madre y hermana con IU. ${ }^{8}$ La diabetes mellitus, la movilidad reducida y los síntomas del tracto urinario (TU) inferior también son frecuentes.

Existen ciertas barreras para el tratamiento, siendo las principales el temor a consultar por sentirse estigmatizadas, el creer que no hay tratamientos efectivos o que ellos son costosos y, por último, el creer que solo hay opciones de manejo invasivas. Debido a eso, solo el $44,9 \%$ de las mujeres consultan por IU, siendo las mujeres con síntomas leves las que menos consultan. ${ }^{9}$
Address for correspondence Mauricio Plata, MD, MSc, FACS, Departamento de Urología, Hospital Universitario Fundación Santa Fe de Bogotá y Facultad de Medicina, Universidad de los Andes, Bogotá D.C., Colombia (e-mail: mplata@uniandes.edu.co).
DOI https://doi.org/ 10.1055/s-0038-1654719. ISSN 0120-789X. eISSN 2027-0119.
Copyright $\odot$ 2018, Sociedad Colombiana License terms de Urología. Publicado por Thieme Revinter Publicações Ltda., Rio de Janeiro, Brazil. Todos los derechos reservados. 


\section{Mecanismos de continencia}

La continencia depende de mecanismos centrales (encéfalo, cerebelo y el cordón espinal) y periféricos (nervios y el sistema de soporte). Durante maniobras de esfuerzo, la continencia es el resultado combinado de la coaptación anatómica pasiva de la uretra y el tono muscular activo del esfínter, pero también del soporte que brindan los tejidos circundantes. La uretra tiene 3 capas: la mucosa, la submucosa esponjosa vascularizada y la capa muscular externa. La mucosa tiene gran importancia, ya que por su configuración y secreciones que aumentan la tensión superficial, se logra la coaptación uretral. $^{10}$ Adicionalmente, cuando la submucosa esponjosa aumenta la perfusión se logra un efecto compresivo. Ese mecanismo explica en parte la IUE en mujeres menopaúsicas, en quienes la disminución del estímulo estrogénico limita la producción de secreciones y proliferación vascular. A su vez, la capa muscular externa está compuesta por fibras circulares y longitudinales que provienen del trígono vesical. Esas fibras son musculares lisas a diferencia del esfínter externo estriado el cual se compone de dos tipos de fibras: las de contracción lenta y las de contracción rápida. Las primeras, crean una contracción continua manteniendo cerrado el esfínter, mientras que las segundas proporcionan la contracción voluntaria. ${ }^{10}$

El soporte anatómico de la uretra, la vejiga y otros órganos pélvicos está dado por los músculos del piso pélvico y las fascias de tejido conectivo. El diafragma pélvico se compone del músculo elevador del ano y los músculos coccígeos. El primero lo forman 3 capas musculares con una abertura natural por donde pasan la uretra, vagina y ano llamado hiato genital. El ligamento pubouretral y la propia pared vaginal son mecanismos de soporte de la uretra femenina que permiten cuando ocurre una maniobra de Valsalva, generar el piso suficiente para que la uretra se cierre y no ocurra pérdida de orina. ${ }^{10}$

\section{Pasado y presente de las teorías de la incontinencia urinaria de esfuerzo}

\section{Teorías anatómicas}

Uno de los primeros autores en describir la apertura del lumen uretral asociado a la IUE, fue Howard Kelly en $1913 .^{11}$ La teoría de Kelly describía que la continencia depende tanto de la uretra como del cuello vesical. Con base en sus observaciones, propuso estrechar el cuello vesical para mejorar la continencia. Él propuso reforzar la pared vaginal anterior y el área a nivel del cuello vesical y la uretra reaproximando las fascias, proporcionando un respaldo contra el que se pudiera comprimir la uretra durante maniobra de Valsalva. La desventaja de esa aproximación es que el resultado dependía de la calidad de tejidos débiles. A principio de los años 20, Victor Bonney describió la pérdida de soporte parauretral como un desplazamiento anormal de la uretra y de la unión uretrovesical por debajo a la sínfisis del pubis, produciendo IU. ${ }^{12}$

\section{Teoría de transmisión de presiones}

Enhörning, siguiendo los lineamientos de Bonney, propuso en 1961 que, además de la pérdida de soporte parauretral, la transmisión desigual de presiones a la uretra y a la vejiga producía incontinencia cuando el cuello vesical y la uretra proximal descendían por debajo del diafragma pélvico; así, la presión vesical superaba la uretral, produciendo escape de orina (-Fig. 1). Se propuso que el llevar la uretra a una posición más abdominal corregiría la IU. Esa teoría fue el fundamento de la aparición de procedimientos retropúbicos como las cirugías de Burch, Marshall-Marchetti-Krantz (MMK) y Tanagho que se mencionan más adelante. ${ }^{13}$

\section{Teorías del déficit esfintérico intrínseco}

En pacientes con cirugía retropúbica fallida, se encontró que la IU estaba frecuentemente asociada a la pobre función de cierre uretral ( - Fig. 2). En estudios de medición de la función uretral

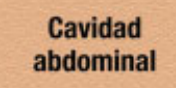

Cavidad
abdominal

Cavidad

abdominal

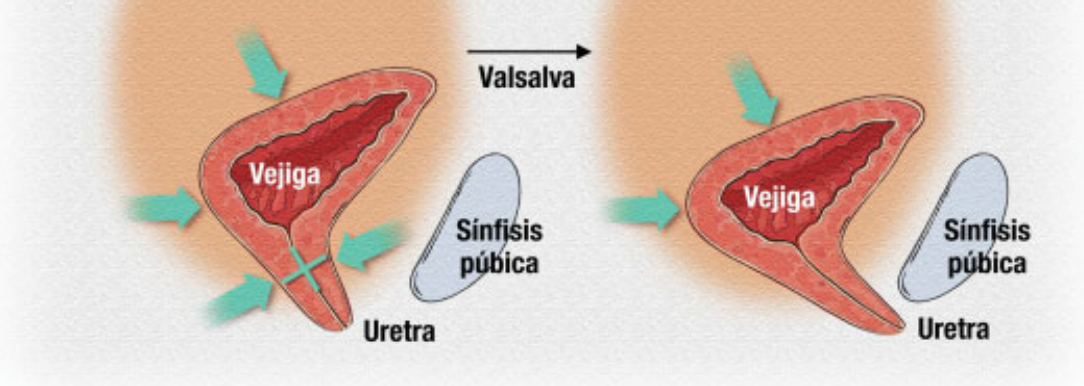

Fig. 1 Teoría de la transmisión de presiones. Durante la maniobra de Valsalva, el cuello vesical y la uretra proximal descienden; si la presión vesical supera la presión uretral, se produce escape de orina. 


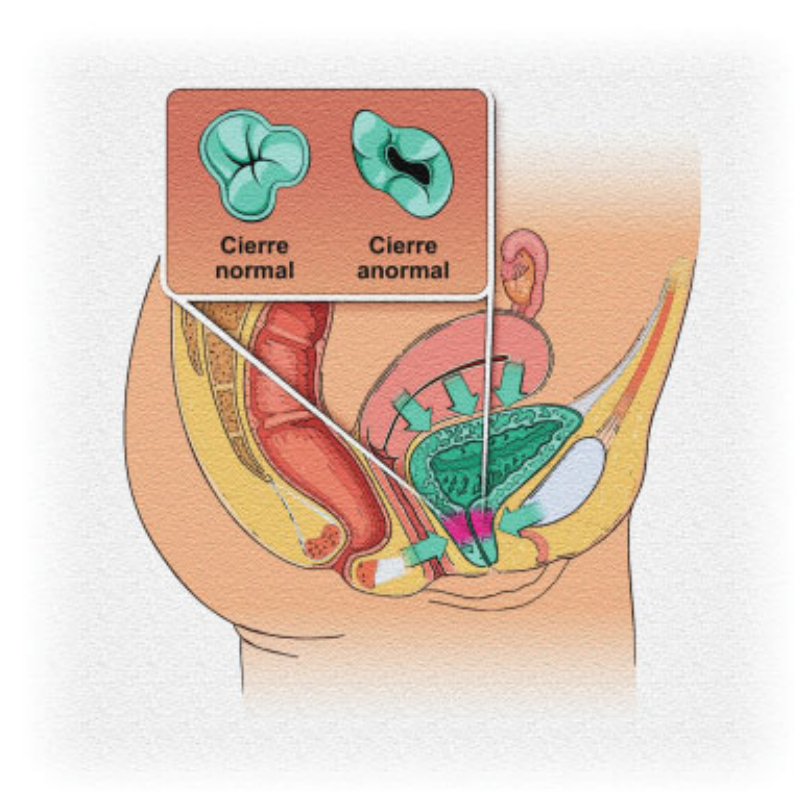

Fig. 2 Teoría del déficit esfintérico intrínseco. Esa teoría explica la imposibilidad de mantener la coaptación de la uretra tanto en reposo como en esfuerzo, lo que conlleva al cierre anormal de la misma.

como la perfilometría uretral, la presión de cierre uretral máxima (PCUM) fue baja, y cuando esos valores eran $<20 \mathrm{cmH}_{2} \mathrm{O}$, fue más frecuente el fracaso de los procedimientos retropúbicos. ${ }^{14} \mathrm{~A}$ eso se le dio el nombre de déficit esfintérico intrínseco (DEI), el cual se refiere a la imposibilidad de mantener la coaptación de la mucosa de la uretra tanto en reposo como en esfuerzo por un compromiso del músculo liso uretral que es ineficiente en generar el cierre de la uretra. Los pacientes que comúnmente tenían esa condición, eran aquellos con antecedente de cirugía pélvica previa, radioterapia, hipoestrogenismo severo que llevaba a que la uretra fuera de reducida movilidad. A partir de estos hallazgos se introdujo el concepto del punto de presión de escape abdominal (ALPP). Él se define como la presión vesical con la cual ocurre escape de orina por aumento de la presión abdominal en ausencia de una contracción involuntaria del detrusor. McGuire y cols. ${ }^{15}$ demostraron que el $76 \%$ de los pacientes con DEI llevados a videourodinamia tienen ALPp $<60 \mathrm{mmH}_{2} \mathrm{O}$. Esa prueba se instauró como la prueba estándar en urodinamia gracias a su simplicidad y confiabilidad. Blaivas demostró que la IUE causada por alteraciones anatómicas o problemas relacionados con el esfínter se puede diagnosticar mediante videourodinamia. ${ }^{16}$ Propuso entonces una clasificación actualmente no utilizada de rutina. En su estudio se clasificó la causa de incontinencia en diferentes tipos siendo los tipos I y II incontinencia por hipermovilidad de la uretra por mal soporte uretral y la llamada tipo III debido a DEI.

\section{Teoría integral y de la hamaca}

La teoría integral de continencia considera básicamente que la IUE, la IUU y las alteraciones del vaciamiento vesical son consecuencia de alteraciones en los elementos de soporte suburetral, como los ligamentos y músculos del piso pélvico, ya que las presiones que se ejerzan sobre esos últimos, se van a traducir en apertura o cierre del cuello vesical y la uretra. Se llama teoría integral porque cuando la uretra está abierta se puede activar de manera temprana el reflejo miccional ocasionando IUU. La uretra media es la que contiene el mecanismo principal de continencia. El daño a los ligamentos pubouretrales que la sostienen, el deficiente soporte de la pared vaginal anterior, y la debilidad de los músculos pubococcígeos son las principales causas de IUE debido a un defecto del soporte suburetral. ${ }^{17}$

La teoría de la hamaca (-Fig. 3), propuesta por DeLancey en el año 1994, explica que la continencia depende de la transmisión de presión al cuello vesical y a la uretra contra la fascia pubocervical y a la pared vaginal anterior. Esa teoría se enfoca principalmente en IUE, mientras que la teoría integral de la continencia toma en cuenta tanto la IUE como IUU. ${ }^{18}$ Existen semejanzas entre esas dos teorías, siendo la integral una interpretación más holística que explica todos los defectos que pueden ocurrir en el piso pélvico, mientras que la teoría de la hamaca está más orientada a explicar el porqué de la IUE.

En resumen, el número de teorías que quieren explicar el porqué de la incontinencia urinaria es alto reflejando la dificultad en el entendimiento y la complejidad de la patología. Para efectos prácticos, en el siglo XXI podemos decir que cada paciente con incontinencia es individual, y en cada uno encontraremos un mayor o menor grado de pérdida de soporte uretral pero también un mayor o menor grado de pérdida de la coaptación uretral o déficit esfintérico. Es nuestro deber como cuidadores de la salud, determinar qué tanto pesa cada uno de ellos y así poder definir la mejor opción terapéutica para cada paciente.

\section{Diagnóstico de la incontinencia urinaria}

\section{Historia clínica}

La historia clínica (HC), es considerada el pilar fundamental del diagnóstico ya que caracteriza correctamente la IU. Hay que recordar que muchas pacientes pueden presentar

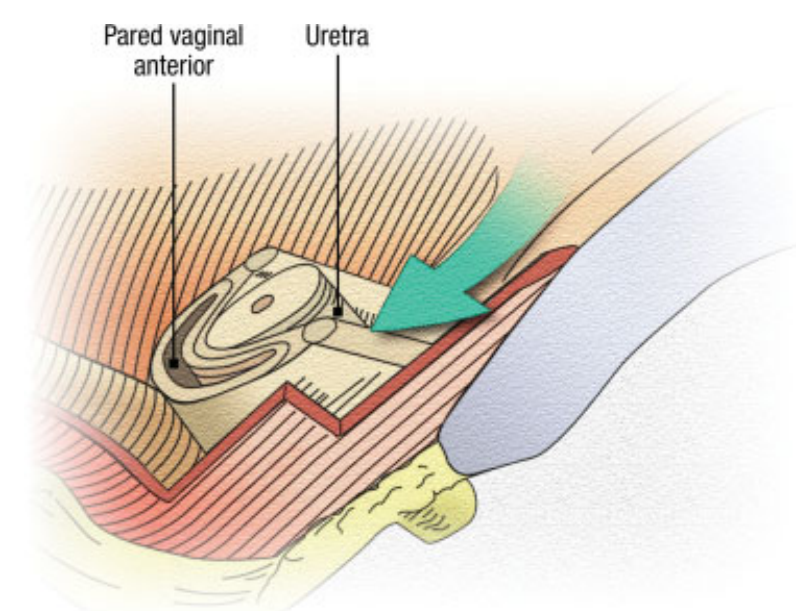

Fig. 3 Teoría de la hamaca. Esa teoría explica que la continencia depende de la transmisión de presión al cuello vesical y a la uretra contra la fascia pubocervical y a la pared vaginal anterior. 
síntomas tanto de esfuerzo como de urgencia urinaria. Se debe tratar de cuantificar la perdida de orina y su relación con la severidad de la incontinencia, describir cómo es el patrón miccional durante el día y la noche, los síntomas obstructivos del TU, la sensación de evacuación incompleta, y el escape de orina con la actividad sexual. La duración de los síntomas es importante, así como conocer si se asocia a eventos como partos, embarazos o cirugías previas.

Otro aspecto a evaluar es el impacto que tiene la IU en la QoL de cada paciente. Eso se hace mediante la aplicación de cuestionarios, índices de síntomas o tablas de desenlaces. Existen cuestionarios de dos tipos: generales de evaluación de QoL como el EuroQoL $5^{19}$ y otros específicos de incontinencia urinaria como el ICIQ (de sus siglas en inglés: International Consultation on Incontinence Questionnaire). ${ }^{20}$ Estudios recientes han demostrado que los formatos basados en internet, parecen ser más aceptados por muchos de los pacientes comparado con las versiones impresas. ${ }^{21}$

\section{Examen físico}

En primer lugar, se debe realizar una inspección y palpación del abdomen en búsqueda de alteraciones vesicales y revisar los genitales externos, evaluando la presencia de lesiones, la distribución del vello púbico y su apariencia general. El meato uretral debe estar normo implantado, sin lesiones o prolapso de mucosa uretral. Se debe observar si existe o no atrofia genital, leucorrea y un examen compartimental para la detección de prolapso de órganos pélvicos (POP). Se debe realizar un tacto vaginal en el cual tanto el cérvix como el útero se inspeccionen, asegurando que su movilidad sea la usual y que no haya un descenso. De igual manera se deben revisar los anexos, asegurando que no haya masas o dolor. Se puede incluir el tacto rectal, teniendo en cuenta el tono del esfínter anal y la presencia de hemorroides o masas rectales.

La posición de la uretra puede ser determinada tanto en reposo como durante maniobras de Valsalva. La prueba del Q-tip nos permite ver, al insertar un hisopo en la uretra, el grado de movilidad. Se considera que ángulos superiores a 30 grados de desplazamiento durante la realización de Valsalva, sugieren hipermovilidad uretral y ángulos $<30^{\circ}$ sugieren uretras fijas (-Fig. 4).

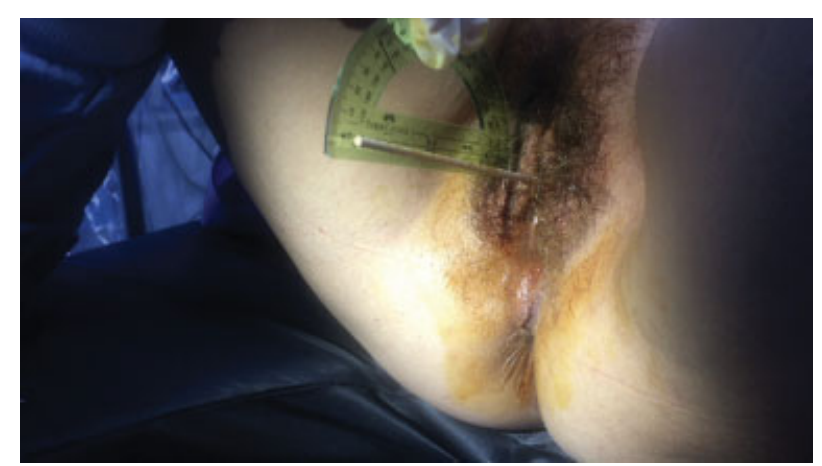

Fig. 4 Test de Q-tip o test del hisopo. Permite ver, al insertar un hisopo en la uretra, el grado de movilidad. Se considera que ángulos superiores a 30 grados de desplazamiento durante la realización de Valsalva sugieren hipermovilidad uretral, y ángulos $<30^{\circ}$ sugieren uretras fijas.

\section{Prolapso de órganos pélvicos}

La valoración de POP se realiza con la paciente acostada y de pie. Existen múltiples clasificaciones de prolapso, siendo las más usadas la de Baden-Walker y el POP-Q (de sus siglas en inglés: Pelvic Organ Prolapse Quantification system; - Fig. 5); ambas toman en cuenta como punto de reparo, la línea himeneal y se mide el grado de protrusión de la pared vaginal anterior, posterior $\mathrm{y}$ apical en reposo y durante maniobra de Valsalva. Esa clasificación permite determinar el estado del prolapso teniendo en cuenta el punto de más declive como referencia. ${ }^{22}$

\section{Examen neurológico}

Es importante determinar la pérdida de sensibilidad perineal y de miembros inferiores, así como la actividad motora. El reflejo bulbocarvenoso, que se realiza al comprimir el glande o el clítoris y buscar contracción del esfínter anal, determina integridad de los niveles sacros S2-S4, el cual está presente en $70 \%$ de las mujeres normales y en el 100\% de los hombres normales. Se puede complementar el examen con un tacto rectal en caso de prolapsos del compartimiento posterior. La sensibilidad perineal y el tono rectal son una buena aproximación para determinar la integridad del segmento S2-S4 responsable de la dinámica miccional.

En la - Tabla 1 se presenta una lista de chequeo con los puntos claves en la HC y en el EF de la mujer con IU.

\section{Exámenes adicionales}

\section{Diario miccional}

Para poder detectar correctamente el tipo de incontinencia, su frecuencia, severidad y síntomas asociados, se puede pedir a la paciente que llene un diario miccional. Ese diario implica que la paciente registre cada vez que consume líquidos y la cantidad en que lo hace. De igual manera debe registrar todos los eventos miccionales, cuantificando el volumen evacuado cada vez. Además, debe registrar las pérdidas urinarias y los síntomas asociados a las mismas. No hay consenso en cuanto al número de días que el paciente debe hacer el diario, pero se considera un estándar el realizarlo durante tres días (-Fig. 6). ${ }^{23}$

\section{Test del protector o pañal (Pad-Test)}

Mediante el pad-test se pueden cuantificar las pérdidas urinarias. No describe el tipo de perdida pero si la cantidad y así la severidad del escape. Se puede realizar durante 1 hora o 24 horas. El pad test de 24 horas es más confiable y la mejor forma para hacerlo es entregarle al paciente un protector 0 pañal previamente pesado el cual debe traer de vuelta a la misma hora el día siguiente. Debe usarlo todo el día y hacer la actividad habitual. El peso registrado se descuenta del peso inicial y esa medida permite determinar la respuesta a tratamientos de una manera semi-objetiva. ${ }^{24}$

\section{Urodinamia}

Es el estudio funcional del TU que evalúa el almacenamiento y evacuación de la orina mediante parámetros fisiológicos. En ella se intenta reproducir los síntomas urinarios con el 


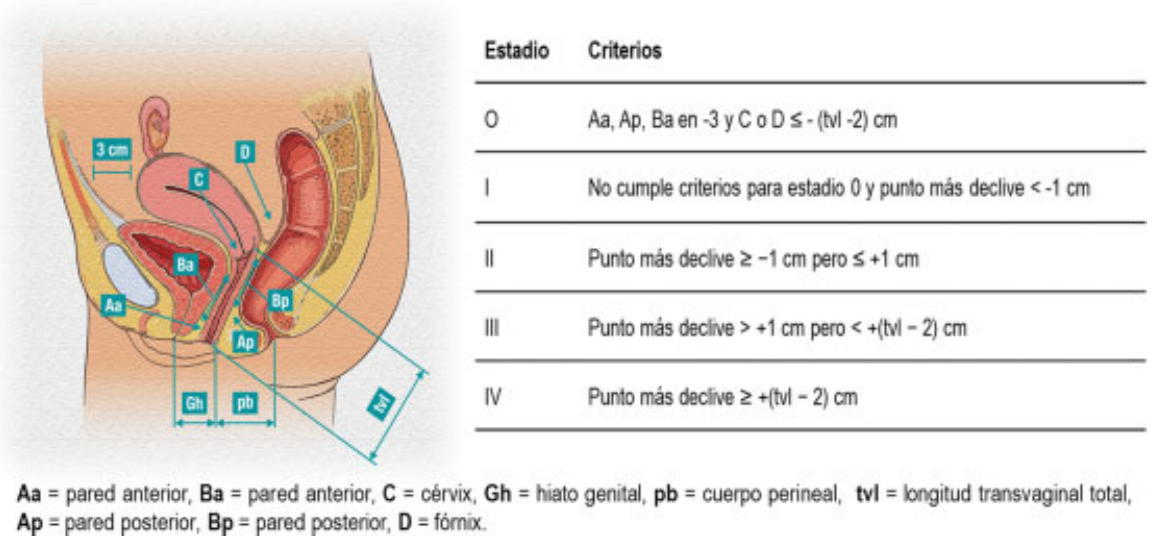

Fig. 5 Clasificación POP-Q. Esa clasifica de 0 a IV el prolapso de órganos pélvicos durante el examen físico en reposo y con maniobra de Valsalva, teniendo en cuenta el punto más en declive como referencia.

fin de identificar su causa y elegir el manejo apropiado (-Fig. 7).

La Sociedad Americana de Urología (AUA) y la Sociedad de Urodinamia, Medicina Pélvica Femenina y de Reconstrucción Urogenital (SUFU) la recomiendan para evaluar la función uretral en mujeres con sospecha de IUE, mujeres a las cuales se planean llevar a manejo quirúrgico de la IU, pacientes con POP de alto grado sin síntomas de IUE, y en pacientes con síntomas de IU posterior a procedimientos que involucren el TU de salida, así como en mujeres con IUM. ${ }^{25}$

Por su parte, la Asociación Europea de Urología (EUA), considera que el estudio urodinámico debe evitarse de rutina en pacientes con recomendación de manejo conservador, aunque se indica su realización en pacientes en los que el resultado de la urodinamia pueda significar cambios en el plan de manejo. ${ }^{20}$
Otras indicaciones reportadas son los pacientes con antecedente de patología neurológica que afecte el TU, cirugía pélvica previa ya sea para corrección de IU o POP (-Tabla 2).

Previo a la uroflujometría libre, es importante pedirle a la paciente que realice maniobra de Valsalva para determinar presencia de escape de orina. Posteriormente, la paciente realiza un flujo urinario no instrumentado el cual puede ser normal o anormal según valores del flujo máximo y se mide el residuo post-miccional. En la urodinamia invasiva, durante la cistometría, se evalúan cinco parámetros: sensación, capacidad cistométrica máxima, distensibilidad (cambio en el volumen/cambio en la presión), actividad del detrusor y función uretral. ${ }^{26}$

Para el estudio de IUE, es de especial importancia la función uretral; para ello se mide la ALPP, capaz de sugerir el tipo de IUE. Se ha visto que mujeres con presiones de

Tabla 1 Lista de chequeo en la evaluación de la incontinencia urinaria

\begin{tabular}{|l|l|}
\hline \multicolumn{2}{|l|}{ Historia Clínica } \\
\hline Caracterización del tipo de incontinencia & Esfuerzo, urgencia, mixta \\
\hline Cuantificación del escape & Número de protectores usados diariamente \\
\hline Determinación del patrón miccional & Frecuencia diurna y nocturna, características de la micción \\
\hline Tiempo & Duración de los síntomas, eventos desencadenantes \\
\hline Prolapso & Sensación de masa vaginal \\
\hline Otras enfermedades & $\begin{array}{l}\text { Neurológicas, demencia, diabetes mellitus, trauma, radioterapia, enfermedad } \\
\text { de Parkinson, enfermedad cerebrovascular }\end{array}$ \\
\hline Historia gineco-obstétrica & Gravidez, paridad, estado hormonal \\
\hline Cirugía pélvica previa & Incontinencia, prolapso, histerectomía \\
\hline Medicamentos & Simpaticomiméticos, simpaticolíticos, antimuscarínicos, diuréticos \\
\hline Examen Físico & \multicolumn{2}{|l|}{} \\
\hline Palpación abdominal & Masas, hernias, globo vesical \\
\hline Genitales externos & Vello púbico, lesiones \\
\hline Meato uretral & Masas, lesiones, atrofia, movilidad con Valsalva y presencia de escape de orina \\
\hline Vagina & $\begin{array}{l}\text { Leucorrea, trofismo, Valsalva } \\
\text { Búsqueda de prolapsos en decúbito y de pie } \\
\text { Tacto: tamaño uterino, posición, movilidad, descenso }\end{array}$ \\
\hline Ano y periné & Tacto rectal \\
\hline
\end{tabular}




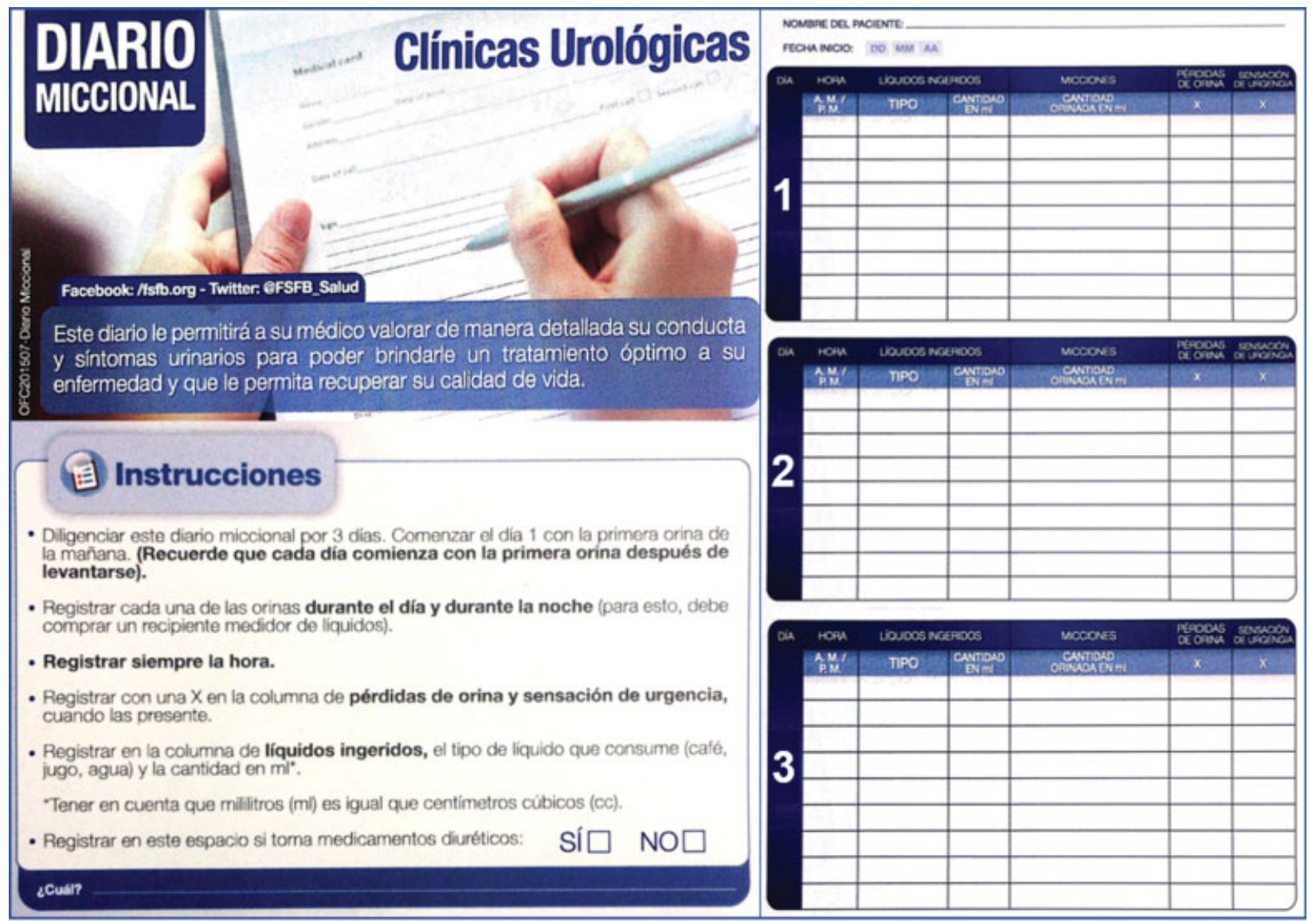

Fig. 6 Diario miccional: Diario miccional para diligenciamiento por el paciente durante 3 días. Se deben diligenciar todos los líquidos ingeridos y los episodios miccionales con hora y volumen. Adicionalmente se deben registrar pérdidas urinarias, sensación de urgencia y uso de diuréticos.

escape $<60 \mathrm{cmH} 20$, se asocian más a la pérdida de coaptación uretral o DEI, mientras que aquellas con presiones $>100 \mathrm{cmH} 2 \mathrm{O}$ tienen menor probabilidad de tenerlo. ${ }^{26}$

El estudio de presión-flujo define la presencia de obstrucción del TU y trastornos de contractilidad del detrusor, lo cual permite elegir el mejor abordaje quirúrgico o la necesidad de procedimientos adicionales.

\section{¿Cuándo sospechar una IUE por DEI?}

Ese punto sigue siendo controversial; sin embargo, una mujer con antecedente de cirugía de incontinencia o prolapso previo, IU severa, pérdida de la motilidad uretral e importante atrofia genital, prueba de Q-tip $<20$ grados y urodinamia con ALPp $<60 \mathrm{cmH} 20$, soportan esa sospecha

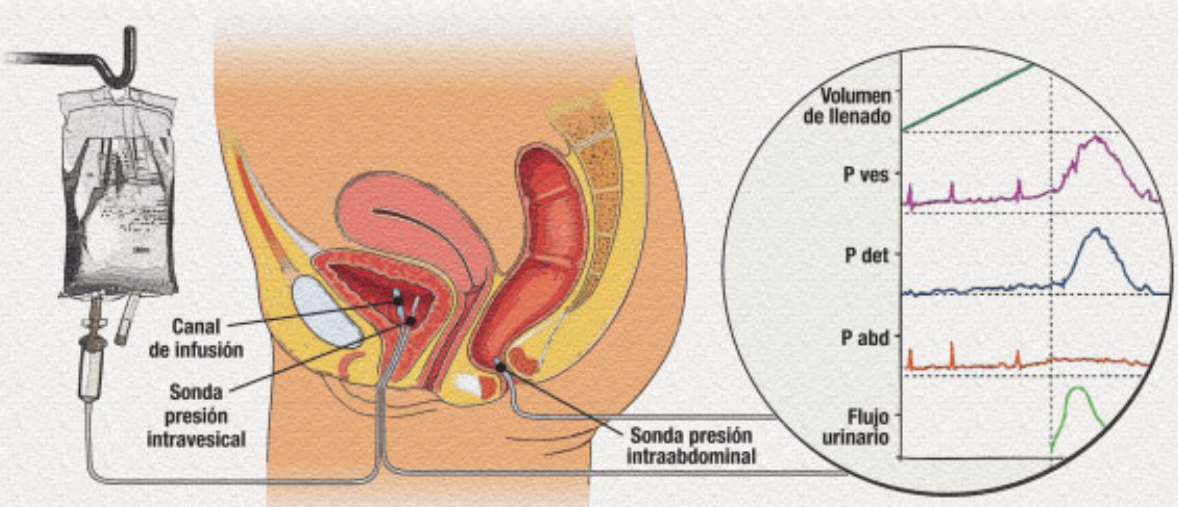

Fig. 7 Urodinamia. Para el estudio de incontinencia urinaria de esfuerzo, es de especial importancia la función uretral, la cual es capaz de sugerir el tipo de incontinencia con el fin de elegir el manejo más apropiado. 
Tabla 2 Indicaciones de urodinamia en incontinencia urinaria de esfuerzo

\begin{tabular}{|l|}
\hline 1. Falla a tratamiento conservador \\
\hline $\begin{array}{l}\text { 2. Evaluación de función uretral para definir el tipo de IUE } \\
\text { cuando se anticipa un procedimiento irreversible. }\end{array}$ \\
\hline $\begin{array}{l}\text { 3. Evaluación de incontinencia oculta en pacientes con } \\
\text { prolapso genital mayor a estado III }\end{array}$ \\
\hline $\begin{array}{l}\text { 4. Pacientes con IUE en quienes se considere manejo invasivo } \\
\text { o irreversible }\end{array}$ \\
\hline $\begin{array}{l}\text { 5. Pacientes con incontinencia urinaria y antecedente de } \\
\text { cirugía pélvica previa }\end{array}$ \\
\hline 6. Patología neurológica que afecte el tracto urinario. \\
\hline
\end{tabular}

Abreviación: IUE, incontinencia urinaria de esfuerzo.

diagnóstica y ayudan a definir el manejo acorde. En algunas mujeres, el componente de pérdida de soporte uretral será mayor que el de pérdida de la coaptación uretral y viceversa por lo cual es útil individualizar cada paciente y, según los hallazgos clínicos y paraclínicos, definir qué tanto componente de DEI o pérdida de soporte uretral puede tener.

\section{Manejo de la incontinencia urinaria de esfuerzo}

Es importante definir si los dos tipos de incontinencia (IUE e IUU) están presentes, y cuánto pesa cada uno de ellos en relación con la sintomatología del paciente. El tipo de incontinencia que predomine es el que va a determinar el énfasis inicial del tratamiento, pero sin olvidar el segundo. El manejo de ambos se divide en diferentes líneas; la primeria incluye cambios de estilos de vida, principalmente la restricción del consumo de líquidos, actividad física moderada y pérdida de peso $(>5 \%$ ) en pacientes obesas, lo cual puede mejorar síntomas como la frecuencia y el volumen evacuado. ${ }^{27}$

Dentro de la primera línea también se incluye la terapia conductual, en la cual se pretende hacer un reentrenamiento vesical. El objetivo es extender el tiempo entre cada micción, buscando recuperar la continencia urinaria al orinar por horario.

\section{Terapia de rehabilitación de piso pélvico}

Los pacientes con IUE, se benefician de los ejercicios de rehabilitación del piso pélvico, pues ellos fortalecen los músculos que lo componen y mejoran la estabilidad uretral y el soporte del cuello vesical. Consisten en ejercicios de contracción y relajación muscular que deben ser supervisados por profesionales entrenados. Al contraer los músculos correctos se logra un pinzamiento de la uretra que aumenta la presión intrauretral, evitando el escape de orina. Adicionalmente, el fortalecimiento del piso pélvico mejora la posición del músculo elevador del ano, evitando escapes de orina con el aumento de la presión abdominal. ${ }^{28}$

Se ha demostrado que la efectividad de la terapia de rehabilitación del piso pélvico aumenta cuando se hace de manera conjunta con biofeedback o bioretroalimentación, que consiste en usar un instrumento para medir la señal biológica generada por los músculos del piso pélvico al contraerse. Él convierte la señal en información visual o auditiva que puede ser interpretada por las pacientes para saber si están realizando correctamente el ejercicio. Los instrumentos usados pueden medir presiones, actividad eléctrica o incluso usar ultrasonido para ver el movimiento de los músculos. ${ }^{29}$ Los ejercicios de Kegel son maniobras útiles, sin embargo, la probabilidad que un paciente los haga adecuadamente cuando se enseñan a hacer de manera verbal es muy baja. Por eso la bioretroalimentación asistida por una fisioterapeuta es la mejor forma de entrenar el piso pélvico.

En resumen, las modificaciones dietarias, hábito miccional y rehabilitación del piso pélvico son tratamientos ya sea como único manejo en el caso de la IUE leve, o complementarios a otras terapias en el caso de la moderada a severa.

\section{Manejo quirúrgico}

El objetivo principal del manejo quirúrgico de la IUE es lograr la continencia sin comprometer la micción, con una tasa aceptable de complicaciones. Muchos procedimientos quirúrgicos no logran ese objetivo. Aproximadamente el $30 \%$ de las cirugías para IUE se realizan en pacientes con IUE recurrente. Esa elevada tasa de falla terapéutica refleja lo poco que se sabe sobre la fisiopatología de la enfermedad. ${ }^{30}$

\section{Abordaje abdominal}

Todos los procedimientos tienen en común la aproximación de la uretra y el cuello vesical al pubis o a ligamentos. Los procedimientos que hacen referencia a esa técnica son el Marshall - Marchetti-Krantz (MMK) y la colposuspensión de Burch. El MMK fue descrito por primera vez en 1949 en un hombre con IU posterior a una prostatectomía. ${ }^{31}$ La descripción original habla de la colocación de suturas vaginales laterales y en el tejido periuretral que se anclan a la sínfisis púbica. Por otra parte, la colposuspensión de Burch descrita en 1961,consiste en aproximar la fascia periuretral al ligamento de Cooper (ileopectíneo) usando tres pares de suturas (-Fig. 8). ${ }^{32}$ Algunos argumentan que la colposuspensión de Burch muestra mejores resultados porque sus suturas elevan mejor los elevadores del ano al estar más laterales que las del MMK. ${ }^{33}$

La colposuspensión fue posteriormente modificada por Tanagho, sumando una aproximación de la pared proximal de la vagina a las paredes laterales de la pelvis. Se buscaba mejorar el soporte y evitar el riesgo de micción disfuncional. ${ }^{34}$ La tasa inicial de éxito fue del 100\%; sin embargo, después de 9 años de seguimiento, se documentó una tasa de enterocele del 8\%. A través del tiempo, variaciones laparoscópicas del procedimiento han sido usadas, pero los resultados parecen no superar la técnica convencional. ${ }^{35}$

Experimentos clínicos aleatorizados han demostrado que la efectividad de la colposuspensión disminuye con el tiempo, pero es altamente dependiente de la forma en la que la incontinencia es medida. El seguimiento a más de 5 años en el estudio SISTEr, que aleatorizó pacientes a cirugía de colposuspensión versus sling pubovaginal como tratamiento de la IUE, mostró que los resultados en cuanto a cura de la incontinencia a 5 años es bajo (24,1\%) con 


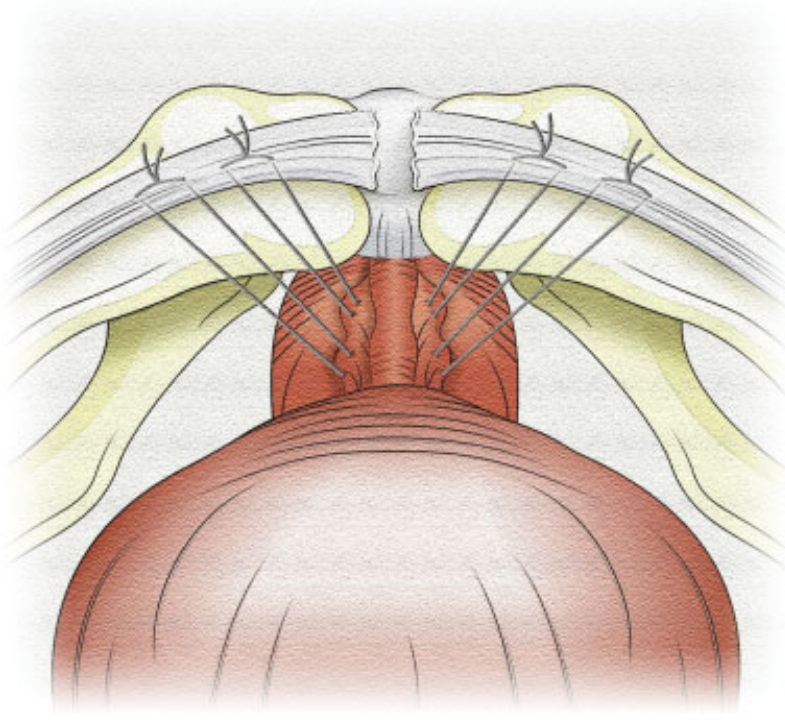

Fig. 8 Colposuspensión de Burch. Consiste en aproximar la fascia periuretral al ligamento de Cooper (ileopectíneo) usando tres pares de suturas.

criterios estrictos de continencia urinaria; sin embargo, la cuantificación de la satisfacción de las pacientes fue alta. ${ }^{30}$ Eso ha generado que las técnicas abdominales hayan caído en desuso en las dos últimas décadas sumado al advenimiento de otras técnicas quirúrgicas.

\section{Suspensión transvaginal}

En el año 1959, Pereyra describió la suspensión transvaginal con aguja. El procedimiento buscaba obtener un soporte del tejido periuretral comparable al del MMK o al de Burch. ${ }^{33}$ Una aguja atraviesa la fascia pericervical y la fija al músculo recto anterior. Stamey propuso una modificación que involucraba un cistoscopio para verificar la posición de las suturas. ${ }^{36}$ Tiempo después, Gittes y Loughlin propusieron el paso a ciegas de la aguja, evitando la incisión vaginal. En 1981, Raz propuso una modificación del método de Pereyra en el que planteaba unir las diferentes capas de la fascia endopélvica y usarlas para obtener soporte. ${ }^{37}$ Gittes en 1987 , usó la misma técnica, con la diferencia de que sus suturas las ubicaba directamente en la pared vaginal. ${ }^{38}$ Se cree que los procedimientos transvaginales tienen el mismo mecanismo que las suspensiones retropúbicas para evitar la IUE. Los resultados a largo plazo palidecen frente a los de los procedimientos retropúbicos, por lo que su uso no se recomienda en este momento.

\section{Cintas libres de tensión}

Descritos por primera vez en 1996 por Ulmsten, son considerados la primera línea de manejo quirúrgico para la IUE, gracias a su naturaleza mínimamente invasiva y sus excelentes resultados a largo plazo. ${ }^{39}$ La tasa inicial de éxito a los 2 años es del $84 \% .^{40}$ Originalmente, el procedimiento consistía en una incisión en la pared vaginal anterior con disección de la mucosa parauretral posterior a la sínfisis púbica. Se introducía una cinta de polipropileno a cada lado de la uretra en dirección cefálica usando dos agujas especialmente diseñadas para perforar la pared abdominal anterior, donde se dejaban los extremos de la malla previa al cierre de las incisiones.

Desde entonces, se han descrito dos técnicas importantes: la cinta transvaginal retropúbica (TVT) ( - Fig. 9A) y la cinta transobturatriz (TOT) ( - Figs. 9B y 9C). La principal diferencia entre ambos procedimientos es la dirección de los vectores, siendo más lateral la de la TOT que la de la TVT.

El mecanismo de acción es el mismo en todos los procedimientos: la cinta da soporte en el punto de la uretra en el que se transmiten las fuerzas de cierre de la misma. Ya que la pared uretral anterior se encuentra fija anteriormente por los ligamentos pubouretrales y la pared uretral posterior carece de soporte, el segmento uretral proximal a la cinta no se mueve en su pared posterior, lo que hace que su luz disminuya con aumentos de presión. Sorprendentemente, la movilidad reducida del cuello vesical no mejora la continencia. Experimentos clínicos aleatorizados han demostrado que las cintas TVT y TOT son equivalentes, con algunas ventajas del abordaje transobturatriz en cuanto a riesgo de sangrado, lesión de víscera hueca o vascular; pero con mayor riesgo de dolor
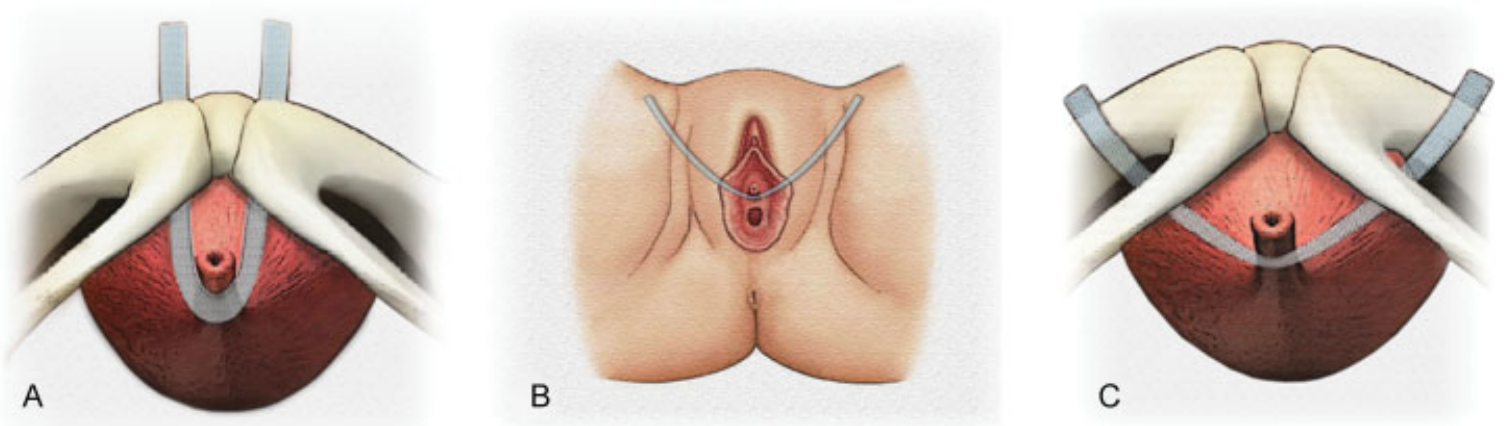

Fig. 9 A. Posición de la cinta transvaginal retropúbica. Se muestra la dirección medial de los vectores, dando soporte a la uretra media. B y C. Posición de la cinta transobturatriz. Se muestra la dirección lateral de los vectores, con un abordaje a través del foramen obturador, distante de las estructuras vasculares, reforzando el ligamento uretropélvico. 
posoperatorio. El seguimiento a largo plazo del estudio TOMUS que evaluó equivalencia entre TVT y TOT demostró a 5 años una pérdida de la equivalencia con una efectividad potencialmente mayor para la primera. ${ }^{41}$

Un área de gran interés es el comportamiento de las cintas libres de tensión en pacientes con sospecha de DEI. Un estudio retrospectivo de Jeon y cols., ${ }^{42}$ demostró la superioridad de las TVT a corto plazo ( 2 años), teniendo una tasa de curación del $86,9 \%$ frente al $34,9 \%$ de la TOT. Sin embargo, a los 7 años de seguimiento, la tasa de curación de la TVT disminuyó a 55,09\%.

Schierlitz y cols., ${ }^{43}$ evaluaron en un experimento clínico, a 164 mujeres con IUE y sospecha de DEI por ALP $p<60 \mathrm{cmH}_{2} \mathrm{O}$ o PCUM $<20 \mathrm{cmH}_{2} \mathrm{O}$. El seguimiento a 3 años demostró que en el grupo de TVT el 21\% de pacientes tenían incontinencia versus $45 \%$ en el grupo de TOT. Adicionalmente, 1 de cada 6 sujetos en el brazo transobturatriz en contra de 1 de 16 en el brazo retropúbico requirió una nueva cirugía.

Se han desarrollado cintas para la uretra media que reducen el riesgo que implica pasar agujas por el espacio obturador o retropúbico. Han sido llamadas cintas de incisión única o mini slings. Se puede ubicar medial o lateral; la primera imita la TOT, mientras que la segunda es más parecida a una retropúbica. Esa cinta evita el paso por zonas de alto riesgo (músculos de los muslos, vasos del espacio obturador, espacio retropúbico), donde existe la posibilidad de lesión a órganos o a estructuras vasculares importantes. Sin embargo, varios estudios aún cuestionan su eficacia a largo plazo. ${ }^{44}$

En la actualidad, las cintas libres de tensión son la técnica de corrección de IU más usada en el mundo. Sin embargo, al tratarse de una cinta sintética, ha generado debate a raíz de complicaciones del uso de ese mismo material en la corrección del POP o las llamadas mallas de corrección de prolapso. Ellas han sido fuertemente criticadas y existe en el momento declaraciones y notificaciones de organismos de regulación internacionales como la FDA advirtiendo sobre las potenciales complicaciones del uso de mallas para el prolapso, no así para las cintas de incontinencia. Sin embargo, en los últimos años, en países como Estados Unidos y el Reino Unido, se ha visto una reducción en el uso de cintas de incontinencia a raíz de ese debate. $^{45}$

En un estudio publicado por Plata y cols., ${ }^{46}$ que evaluó las tendencias en el manejo de POP en Latinoamérica, se vio que el $41 \%$ de 529 urólogos y ginecólogos encuestados consideraban que las advertencias de la FDA sobre el uso de mallas de prolapso impactaron negativamente en el uso de las cintas de incontinencia. Hasta este momento, ninguna entidad reguladora ha hecho un pronunciamiento formal al respecto, pero la AUGS (Sociedad Americana de Uroginecología), considera que la restricción en el uso de las cintas para IUE no está soportada por ninguna organización, incluyendo la FDA. ${ }^{47}$

\section{Cintas autólogas fijas}

Hace más de un siglo se describió por primera vez la idea de sostener la uretra con una cinta de tejido, siendo Aldridge el primero en popularizarlo en 1942. Las descripciones más antiguas involucran los músculos gracilis y piramidalis. En 1978, McGuire usó un injerto de la fascia del recto para elevar el cuello vesical, otorgando cierta obstrucción de la uretra durante las altas presiones de la maniobra de Valsalva. ${ }^{48}$

Los slings pubovaginales integran una aproximación retropúbica y transvaginal. El injerto debe medir aproximadamente $8 \times 2 \mathrm{~cm}$, sin importar si se toma de la fascia lata o de la fascia del recto. En posición de litotomía, se coloca el injerto en forma de $\mathrm{U}$, rodeando el cuello vesical y fijándolo a la pared abdominal. Dada su eficacia en casos de IU con sospecha de DEI, el uso de esos procedimientos se volvió muy popular en los años 90 . Sin embargo, el riesgo de obstrucción del TU existe, lo cual hace que sea una técnica para casos de IUE compleja. Una serie retrospectiva de Chaikin y cols., ${ }^{49}$ en 251 mujeres mostró cura subjetiva del $92 \%$ con seguimiento a 3 años y retención urinaria permanente en el $2 \%$.

\section{Cintas ajustables sintéticas}

Desde hace más de dos décadas se han utilizado las cintas ajustables como opción de manejo de la IU compleja con sospecha de DEI. Tiene la ventaja de ser un procedimiento mínimamente invasivo, pues la cinta es más pequeña, ofreciendo una menor superficie de erosión. Se pueden ajustar fácilmente en el consultorio y el riesgo de obstrucción es menor. En el caso que la paciente tenga síntomas sugestivos de obstrucción urinaria, ésa puede ser fácilmente revertida en el consultorio.

La evidencia disponible corresponde a estudios retrospectivos; sin embargo, los resultados han sido reproducidos en varios centros con tasas de éxito de más del $80 \%{ }^{50}$

\section{Agentes abultantes}

La primera descripción de inyección de agentes abultantes ocurrió en 1938 e incluía la inyección de morruato de sodio. En la década de los 90, la inyección de esos agentes era el procedimiento más frecuentemente utilizado para la corrección de IUE. Sin embargo, un ensayo clínico multicéntrico no logró demostrar equivalencia de Zuidex (dextranómero/ácido hialurónico) con inyección transuretral de colágeno en mujeres con ISD. Adicionalmente se documentó una tasa muy elevada de complicaciones, principalmente pseudo-abscesos estériles. ${ }^{51}$

A raíz de sus resultados mediocres y el nacimiento de nuevas y mejores alternativas en el manejo de la IUE, no se considera la técnica de elección. Sin embargo, se pueden considerar como una opción de tratamiento por su naturaleza mínimamente invasiva; además, son bien tolerados y benéficos a corto plazo. El paciente ideal para un agente abultante de uretra sería aquél que es mal candidato para cirugía, pacientes anticoagulados, 0 aquellos que no deseen una intervención quirúrgica. Se debe advertir sobre la posibilidad de re-inyección, la limitada tasa de éxito y la corta duración del efecto. 


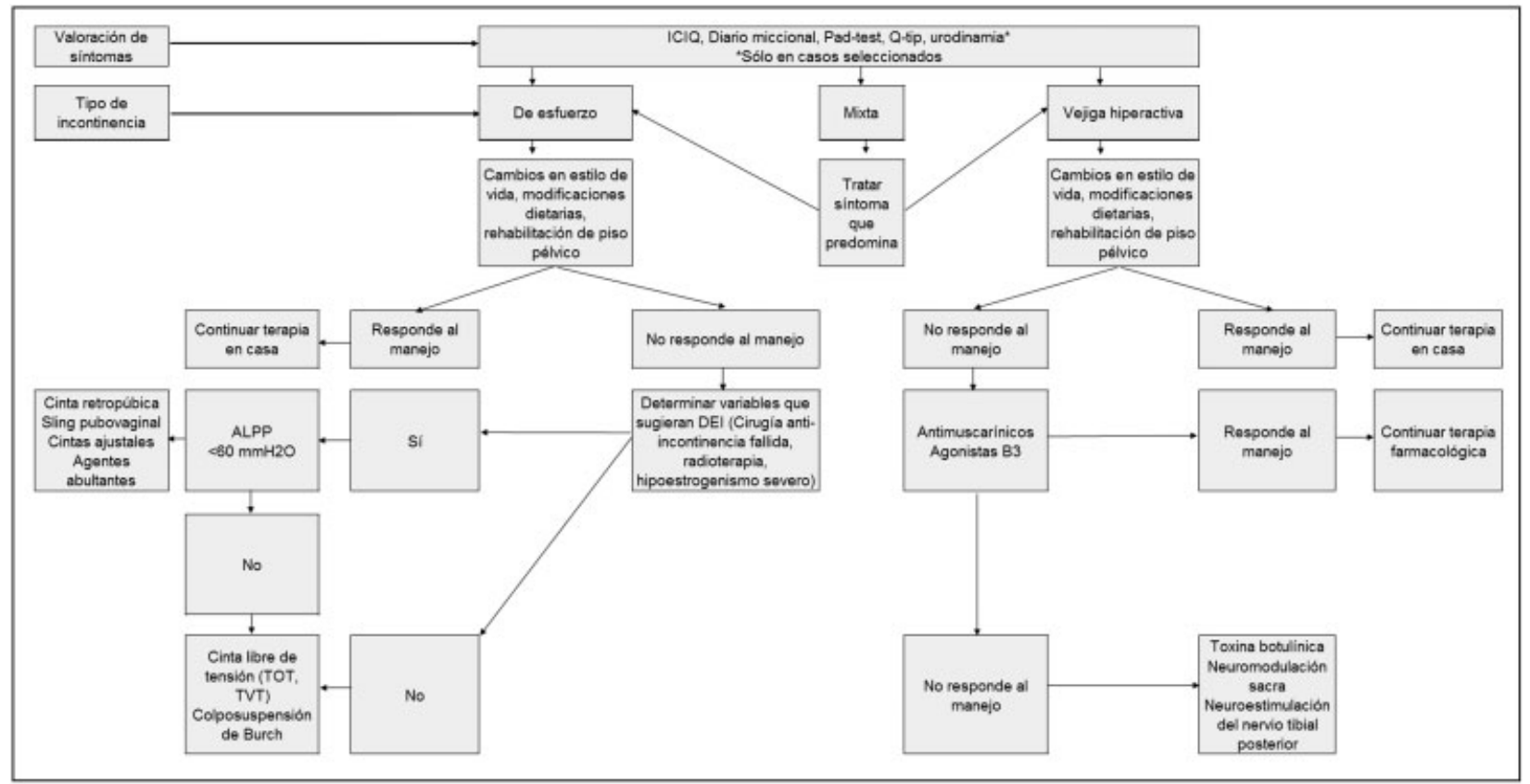

Fig. 10 Algoritmo de manejo de incontinencia urinaria.

\section{Conclusiones}

El pilar del manejo de la IU es una adecuada HC y EF. Pruebas adicionales como el diario miccional, pad-test y algunas pruebas como el Q-tip o la simulación del efecto de una intervención, pueden se maniobras útiles. La urodinamia tiene un rol en la evaluación de la incontinencia compleja o en el paciente en que se anticipe un procedimiento quirúrgico irreversible. La escogencia del mejor tratamiento, depende de varios factores como son: las características propias del paciente, la severidad y tipo de IUE, la disposición a asumir el riesgo de una cirugía y las mejores herramientas disponibles en cada centro (-Fig. 10).

\section{Bibliografía}

1 Abrams P, Cardozo L, Fall M, et al; Standardisation Sub-Committee of the International Continence Society. The standardisation of terminology in lower urinary tract function: report from the standardisation sub-committee of the International Continence Society. Urology 2003;61(01):37-49

2 Thomas TM, Plymat KR, Blannin J, Meade TW. Prevalence of urinary incontinence. BMJ 1980;281(6250):1243-1245

3 Norton PA, MacDonald LD, Sedgwick PM, Stanton SL. Distress and delay associated with urinary incontinence, frequency, and urgency in women. BMJ 1988;297(6657):1187-1189[Internet]

4 Guarisi T, Pinto Neto AM, Osis MJ, Pedro AO, Paiva LHC, Faúndes A. Incontinência urinária entre mulheres climatéricas brasileiras: inquérito domiciliar. Rev Saude Publica 2001;35(05):428-435

5 Plata M, Bravo-Balado A, Robledo D, et al. Prevalence of Lower Urinary Tract Symptoms and Overactive Bladder in Men and Women over 18 Years Old: The Colombian Overactive Bladder and Lower Urinary Tract Symptoms (COBaLT) Study [unpublished]. Accepted for publication in Neurourol Urodynam, January, 2018.

6 Abdo CHN, Oliveira WM Jr, Moreira ED, Fittipaldi JAS. Perfil sexual da população brasileira: resultados do Estudo do Comportamento Sexual (ECOS) do Brasileiro. Rev Bras Med 2002;(59):250-257
7 Viktrup L, Lose G, Rolff M, Barfoed K. The symptom of stress incontinence caused by pregnancy or delivery in primiparas. Obstet Gynecol 1992;79(06):945-949

8 Hannestad YS, Lie RT, Rortveit G, Hunskaar S. Familial risk of urinary incontinence in women: population based cross sectional study. BMJ 2004;329(7471):889-891

9 Herzog AR, Fultz NH, Normolle DP, Brock BM, Diokno AC. Methods used to manage urinary incontinence by older adults in the community. J Am Geriatr Soc 1989;37(04):339-347

10 Kalejaiye O, Vij M, Drake MJ. Classification of stress urinary incontinence. World J Urol 2015;33(09):1215-1220

11 Kelly HA. Incontinence of urine in women. Urol Cutaneous Rev 1913;17(06):291-293

12 Bonney V. On Diurnal Incontinence of Urine in Women. BJOG An Int J Obs Gynaecol. 1923;30(03):358-365

13 Enhorning G. Simultaneous recording of intravesical and intraurethral pressure. A study on urethral closure in normal and stress incontinent women. Acta Chir Scand Suppl 1961 (Suppl 276):1-68

14 Sand PK, Bowen LW, Panganiban R, Ostergard DR. The low pressure urethra as a factor in failed retropubic urethropexy. Obstet Gynecol 1987;69(3 Pt 1):399-402

15 McGuire EJ, Fitzpatrick CC, Wan J, et al. Clinical assessment of urethral sphincter function. J Urol 1993;150(5 Pt 1):1452-1454

16 Blaivas JG, Olsson CA. Stress incontinence: classification and surgical approach. J Urol 1988;139(04):727-731

17 Petros PE, Ulmsten UI. An integral theory of female urinary incontinence. Experimental and clinical considerations. Acta Obstet Gynecol Scand Suppl 1990;153:7-31

18 DeLancey JO. Structural support of the urethra as it relates to stress urinary incontinence: the hammock hypothesis. Am J Obstet Gynecol 1994;170(06):1713-1720, discussion 1720-1723

19 Leidl R, Reitmeir P. An Experience-Based Value Set for the EQ-5D5L in Germany. Value Health 2017;20(08):1150-1156

20 Lucas MG, Bedretdinova D, Bosch JLHR, et al. Guidelines on Urinary Incontinence. Eur Assoc Urol 2014;1:120

21 Parnell BA, Dunivan GC, Connolly A, Jannelli ML, Wells EC, Geller EJ. Validation of web-based administration of the Pelvic Organ Prolapse/Urinary Incontinence Sexual Function Questionnaire 
(PISQ-12). Int Urogynecol J Pelvic Floor Dysfunct 2011;22(03): 357-361

22 Centers for Medicare and Medicaid Services. 1997 documentation guidelines for evaluation and management services. 1997

23 Addla S, Adeyoju A, Neilson D. Assessment of reliability of 1-day, 3-day and 7-day frequency volume charts. Eur Urol Suppl -.;3 (02):130

24 Walsh JB, Mills GL. Measurement of urinary loss in elderly incontinent patients. A simple and accurate method. Lancet 1981;1(8230):1130-1131

25 Winters JC, Dmochowski RR, Goldman HB, et al. Adult Urodynamics: AUA/SUFU Guideline. Am Urol Assoc.; 2012:1-30

26 Plata M, Torres L. El estudio urodinámico. Urol Colomb. 2014;23 (02):128-139

27 Gormley EA, Lightner DJ, Faraday M, Vasavada SP; American Urological Association; Society of Urodynamics, Female Pelvic Medicine. Diagnosis and treatment of overactive bladder (nonneurogenic) in adults: AUA/SUFU guideline amendment. J Urol 2015;193(05):1572-1580

28 Hay J, Berghmans B, Burgio K, Dumoulin C, Hagen S, Moore K. Adult Conservative Management. Committee 12. Int Cont Soc 2009:1025-1118

29 Herderschee R, Hay-Smith ECJ, Herbison GP, Roovers JP, Heineman MJ. Feedback or biofeedback to augment pelvic floor muscle training for urinary incontinence in women: shortened version of a Cochrane systematic review. Neurourol Urodyn 2013;32(04): 325-329

30 Albo ME, Richter HE, Brubaker L, et al; Urinary Incontinence Treatment Network. Burch colposuspension versus fascial sling to reduce urinary stress incontinence. N Engl J Med 2007;356(21): 2143-2155

31 Marshall VF, Marchetti AA, Krantz KE. The correction of stress incontinence by simple vesicourethral suspension. Surg Gynecol Obstet 1949;88(04):509-518

32 Burch JC. Cooper's ligament urethrovesical suspension for stress incontinence. Nine years' experience-results, complications, technique. Am J Obstet Gynecol 1968;100(06):764-774

33 Pereyra AJ. A simplified surgical procedure for the correction of stress incontinence in women. West J Surg, Obstet Gynecol 1959; 67(04):223-226

34 Tanagho EA. Colpocystourethropexy: the way we do it. J Urol 1976;116(06):751-753[Internet]

35 Su TH, Wang KG, Hsu CY, Wei HJ, Hong BK. Prospective comparison of laparoscopic and traditional colposuspensions in the treatment of genuine stress incontinence. Acta Obstet Gynecol Scand 1997;76(06):576-582

36 Stamey TA. Endoscopic suspension of the vesical neck for urinary incontinence. Surg Gynecol Obstet 1973;136(04):547-554

37 Raz S. Modified bladder neck suspension for female stress incontinence. Urology 1981;17(01):82-85

38 Gittes RF, Loughlin KR. No-incision pubovaginal suspension for stress incontinence. J Urol 1987;138(03):568-570
39 Ulmsten U, Henriksson L, Johnson P, Varhos G. An ambulatory surgical procedure under local anesthesia for treatment of female urinary incontinence. Int Urogynecol J Pelvic Floor Dysfunct 1996;7(02):81-85, discussion 85-86

40 Ward KL, Hilton P; UK and Ireland TVT Trial Group. A prospective multicenter randomized trial of tension-free vaginal tape and colposuspension for primary urodynamic stress incontinence: two-year follow-up. Am J Obstet Gynecol 2004;190(02):324-331

41 Richter HE, Albo ME, Zyczynski HM, et al; Urinary Incontinence Treatment Network. Retropubic versus transobturator midurethral slings for stress incontinence. N Engl J Med 2010; 362(22):2066-2076

42 Jeon M-J, Jung H-J, Chung S-M, Kim S-K, Bai S-W. Comparison of the treatment outcome of pubovaginal sling, tension-free vaginal tape, and transobturator tape for stress urinary incontinence with intrinsic sphincter deficiency. Am J Obstet Gynecol 2008;199(01): 76.e1-76.e4

43 Schierlitz L, Dwyer PL, Rosamilia A, et al. Effectiveness of tensionfree vaginal tape compared with transobturator tape in women with stress urinary incontinence and intrinsic sphincter deficiency: a randomized controlled trial. Obstet Gynecol 2008; 112(06):1253-1261

44 Mostafa A, Lim CP, Hopper L, Madhuvrata P, Abdel-Fattah M. Single-incision mini-slings versus standard midurethral slings in surgical management of female stress urinary incontinence: an updated systematic review and meta-analysis of effectiveness and complications. Eur Urol 2014;65(02):402-427

45 Chapple CR, Raz S, Brubaker L, Zimmern PE. Mesh sling in an era of uncertainty: lessons learned and the way forward. Eur Urol 2013; 64(04):525-529

46 Plata MAM, Bravo-Balado A, Robledo D, et al. Trends in pelvic organ prolapse management in Latin America. Neurourol Urodyn 2017;Doi: http://doi.wiley.com/10.1002/nau.23392

47 American Urogynecologic Society Board of Directors. Position statement on restriction of surgical options for pelvic floor disorders: the American Urogynecologic Society Board of Directors. Female Pelvic Med Reconstr Surg 2013;19(04): 199-201

48 Mcguire EJ, Lytton B. Pubovaginal sling procedure for stress incontinence. J Urol 1978;119(01):82-84

49 Chaikin DC, Rosenthal J, Blaivas JG. Pubovaginal fascial sling for all types of stress urinary incontinence: long-term analysis. J Urol 1998;160(04):1312-1316

50 Plata M, Robledo D, Bravo-Balado A, et al. Effectiveness of adjustable slings (Remeex system ${ }^{\mathrm{TM}}$ ) in women with stress urinary incontinence due to intrinsic sphincter deficiency. Eur Urol Suppl 2017;16(03):e1506-e1507

51 Lightner D, Rovner E, Corcos J, et al; Zuidex Study Group. Randomized controlled multisite trial of injected bulking agents for women with intrinsic sphincter deficiency: mid-urethral injection of Zuidex via the Implacer versus proximal urethral injection of Contigen cystoscopically. Urology 2009;74(04):771-775 